\title{
Measurement of 3rd order spherical aberration coefficient for scanning transmission electron microscopy
}

\author{
Y. Kotaka, ${ }^{*}$ T. Yamazaki, ${ }^{* *}$ K. Watanabe, ${ }^{* * *}$ Y. Kikuchi,**** N. Nakanishi, ${ }^{* *}$ and I \\ Hashimoto** \\ * Materials \& Material Engineering Laboratories, Fujitsu Lab. Ltd., Kanagawa, 243-0197, Japan \\ ** Department of Physics, Tokyo University of Science, Tokyo, 162-8601, Japan \\ *** Tokyo Metropolitan College of Technology, Tokyo, 140-0011, Japan \\ **** MIRAI Project, ASET, Tsukuba, 305-8569, Japan
}

Recently, as the technique for improvement of the spatial resolution of HRTEM and highresolution scanning transmission electron microscopy (STEM), the developments of an spherical aberration corrector ( $C_{s}$ corrector) have been positively performed and succeeded by many researchers in the whole world over. In this case, it is necessary to exactly measure the uncorrected and corrected $C_{s}$. Conventionally, the $C_{s}$ has been determined by analyzing a power spectrum image of high resolution image of an amorphous film [1, 2]. However, use of the power spectrum image becomes problematic when the spherical aberration coefficient is low $(<0.5 \mathrm{~mm})$ [3]. Furthermore, it is very difficult to obtain the high qualitative BF STEM image of an amorphous structure because of the low level of probe intensities and the low signal-to-noise ratio. In fact, the estimated values include a very large percentage error. On the other hand, a ronchigram has been expected as another promising technique. In this paper, the measurement taken using crystalline material along lowerindex zone axis is suggested by expanding the method for $C_{s}$ measurement suggested by Liu and Cowley [4].

Czochralski-grown [100]-orientated $p$-type $\mathrm{Si}$ wafer as a specimen for ronchigram observations was prepared because it is easy to make the thin specimen for TEM observation. TEM observations were performed with a JEM-2010F TEM/STEM, operated at $200 \mathrm{keV}$, equipped with a pole piece designed for $C_{s}=1.0 \mathrm{~mm}$. The ronchigrams were obtained by entirely removing the objective aperture.

In order to present how to measure $C_{s}$, the simulated ronchigram at $C_{s}=1.0 \mathrm{~mm}, \Delta f=-450 \mathrm{~nm}$ and $t=20 \mathrm{~nm}$ is displayed in Fig. 1(a). It should be noted that there are characteristic fringes along Kikuchi bands. The corresponding intensity line profile between two white arrow heads shown in Fig. 1(a) is plotted in Fig. 1(b) by solid line. In order to find the origin of this fringe, the ronchigram simulation was performed using three beam condition with 000, 111, and -1-1-1 reflections. The corresponding line profile is shown in Fig. 1(b) by dashed line. The both period and shape under three beam condition are almost the same as those of many beam condition. Therefore, it is concluded that these characteristic fringes are formed by the interactions between 000, 111 and -1-11 reflections.

The turning points of intensity line profiles along $K_{y}$ direction are labeled by $n=1,2, \ldots$, as shown in Fig. 1(b), so that we can get the $K_{y}{ }^{2}-n$ plot. Under three beam condition constituted by systematic reflections, the relationship between $n$ and $K_{y}$ is given by

$$
n=\lambda^{3} C_{s} g^{2} K_{y}^{2}+\lambda g^{2} \Delta f+(1 / 2) \lambda^{3} C_{s} g^{4}+D,
$$

where $\lambda$ is the wave length, $D$ the constant value. As a result, the $C_{s}$ is determined from the sloop of the straight line. Figure 1(c) shows the $K_{y}{ }^{2}-n$ plots obtained from the dynamical simulation of ronchigrams using many beam and three beam conditions, respectively. Both results show a good 
agreement. The $C_{s}$ values measured under these two conditions were $0.998 \mathrm{~mm}$ and $1.000 \mathrm{~mm}$, respectively.

Figures 2(a)-(c) show the experimental ronchigrams at $\Delta f=-300,-400$ and $-500 \mathrm{~nm}$, respectively. In this case, the defocus values displayed in CRT monitor are shown. The values of $C_{s}$ measured at the respective defocus values are shown together with. It is confirmed that the present method is independent of the defocus values. The $C_{s}$ is estimated to be $1.099 \pm 0.005 \mathrm{~mm}$, which was obtained by averaging $C_{s}$ over all conditions. In this way, the error limit is drastically improved compared with the power spectrum analysis and ronchigrams of minerals.

\section{References}

[1] John C. H. Spence, High-Resolution Electron Microscopy (Monographs on the Physics and Chemistry of Materials), Oxford University Press, UK, 2003.

[2] K. Wang, et al., Ultramicroscopy 40 (1992) 139.

[3] E. M. James, et al., J. Electron Microsc. 47 (1998) 561.

[4] J. A. Liu and J. M. Cowley, Ultramicroscopy 19 (1986) 31.

[5] This research was partially supported by the Ministry of Education, Science, Sports and Culture, Grant-in-Aid for Scientific Research (C), 15560024, 2003.
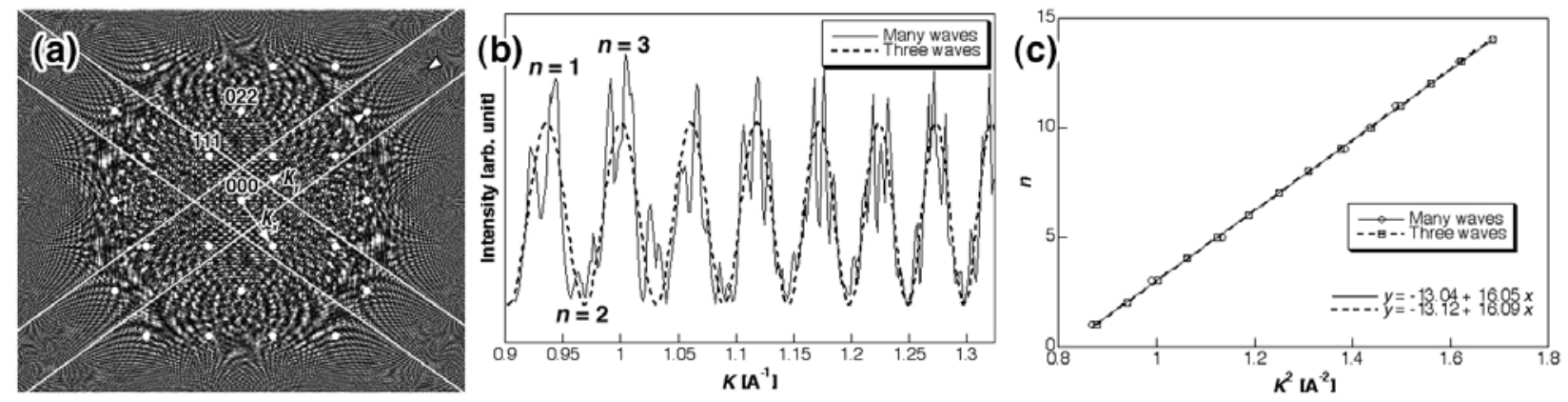

FIG. 1. Schematic drawing of method for measurement of spherical aberration. (a) Simulated ronchigram at $\Delta f=-450 \mathrm{~nm}$ and $t=20 \mathrm{~nm}$. The white dots and white lines express the positions of Bragg reflections and Kikuchi bands of (111) reflections. (b) Intensities profiles by many beam and three beam conditions, and (c) $K^{2}-n$ plots of the corresponding intensities line profiles.
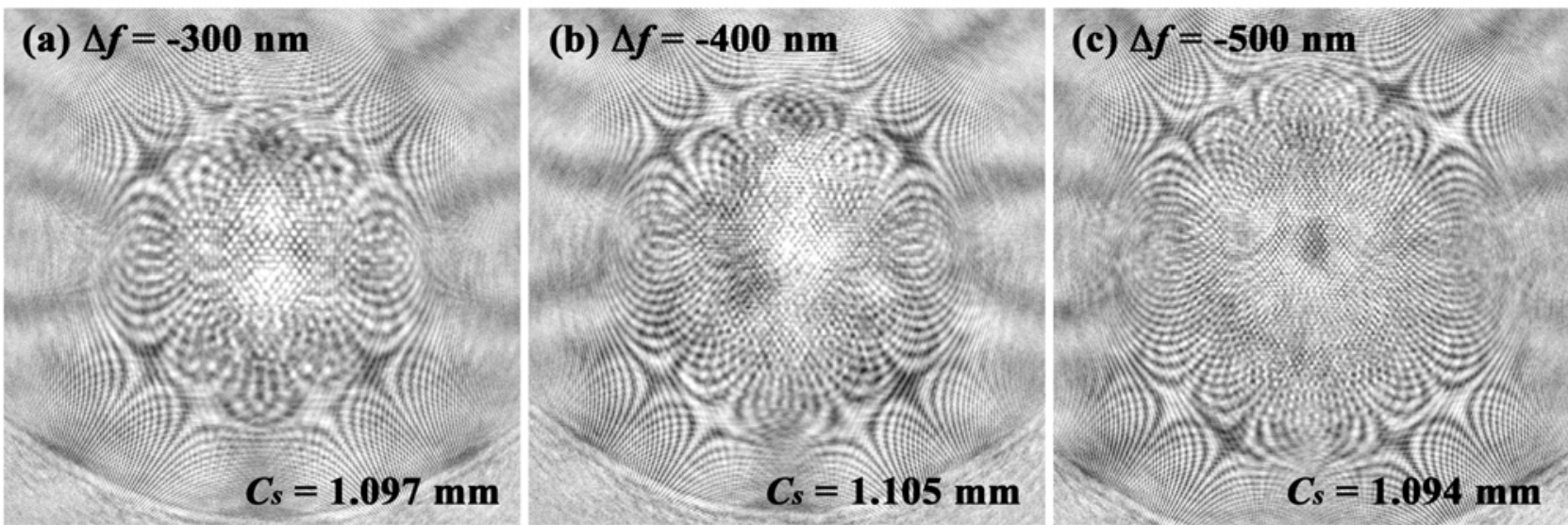

FIG. 2. (a)-(c) Experimental ronchigrams taken at respective defoci together with the measured the $C_{s}$ values. 